\title{
A Multiobjective Optimization Method for Designing $M$-Channel NPR Cosine Modulated Filter Bank for Image Compression
}

\author{
Anamika Jain, Aditya Goel \\ Department of Electronics and Communication Engineering, Maulana Azad National Institute of Technology, \\ Bhopal, India \\ Email: ajain bpl@yahoo.co.in, adityagoel2@rediffmail.com
}

Received 6 February 2015; accepted 24 February 2015; published 27 February 2015

Copyright @ 2015 by authors and Scientific Research Publishing Inc.

This work is licensed under the Creative Commons Attribution International License (CC BY). http://creativecommons.org/licenses/by/4.0/

c) (i) Open Access

\section{Abstract}

This paper proposes a method to design multichannel cosine modulated filter bank for image compression using multiobjective optimization technique. The design problem is a combination of stopband residual energy, least square error of the overall transfer function of the filter bank, coding gain with dc leakage free condition as constraint. The proposed algorithm uses Nondominated Sorting Genetic Algorithm (NSGA) to minimize the mutually contradictory objective function by minimizing filter tap weights of prototype filter. The algorithm solves this problem by searching solutions that achieve the best compromise between the different objectives criteria. The performance of this algorithm is evaluated in terms of coding gain and peak signal to noise ratio (PSNR). Simulation results on different images are included to illustrate the effectiveness of the proposed algorithm for image compression application.

\section{Keywords}

Cosine Modulated Filter Bank, Near Perfect Reconstruction (NPR), Non-Dominated Sorting Genetic Algorithm (NSGA), Peak Signal to Noise Ratio (PSNR), Pseudo Quadrature Mirror Filter (PQMF) Bank

\section{Introduction}

Filter banks find application in various fields of signal, image and video processing for subband/transform coding [1]-[3]. In subband coding of images, filter banks are used to decompose the input image into several subimages in terms of different frequency bands. These subimages are then quantized and decoded to have compres- 
sion with least distortion in reconstructed image quality. To get better resolution, decomposition in $\mathrm{M}$ bands can be achieved in one/two stage via $M$-channel filter bank or via repetitive use of wavelets ( 2 channel filter bank) in five/six stages [4]-[6]. Thus wavelet results in increased delay with nonuniform band decomposition. Filter banks are the most important component in image processing as the quality of the reconstructed image depends mainly on analysis/synthesis filter bank structure. Filter banks are designed as a transform to provide maximum energy compaction and decorrelation. To fulfil both the requirements, filter bank should have least stopband energy with least square error in overall transfer function. However, stopband attenuation increases if the perfect reconstruction condition is relaxed [7]. Amongst various types of transforms/filter banks cosine modulated, filter banks are the most popular choice as the designing and implementation both are easy as compared to others [8] [9]. Many approaches have been proposed for the design of 2 channel filter bank and $M$-channel CMFB filter banks with perfect and near perfect reconstruction condition since long time [10]-[11]. These approaches are based on the standard constrained, unconstrained, iterative least square optimization techniques which are in general complex, depend on the starting point and lead to local solution and may not fulfill all the objectives criterion. Further, quantization and coding of the transformed coefficients are achieved via progressive block based embedded image coder.

In this paper, an approach based on multiobjective optimization i.e. non-dominated sorting genetic algorithm [12] [13] is used to design $M$-channel uniform near perfect reconstruction Cosine modulated filter banks with coding gain given the highest priority amongst other objectives and Shapiro's EZW coder with modifications suitable for block based transform coding is used for coding/decoding.

The organization of the paper is as follows: in Section 2, a relevant brief analysis of the pseudo cosine modulated filter bank is given. In Section 3 optimization problem is formulated. A brief overview of multiobjective optimization algorithm is explained in Section 4. In Section 5, design examples (cases) and results are presented with their application to image coding and conclusions are drawn in Section 6.

\section{Problem Description}

\subsection{Design of Cosine Modulated Filter Bank}

A typical $M$-band filter bank is shown in Figure 1. Input signal $(x[n])$ is decomposed into $M$ subband by analysis filters $\left(H_{0}(z) \cdots H_{M-1}(z)\right)$ in transmitter side, these subbands are then decimated by a factor of $M$. At the receiver side, these subband are up sampled and recombined to get the reconstruction output $y[n]$ with the aid of synthesis filters $\left(F_{0}(z) \cdots F_{M-1}(z)\right)$. When these analysis /synthesis filters are cosine modulated version of a low pass prototype filter, then these filter banks are termed as cosine modulated filter banks. Based on input/output relationship of filter bank the reconstruction output is expressible as [14]

$$
\hat{X}(z)=\frac{1}{M} T_{0}(z) X(z)+\frac{1}{M} \sum_{k=0}^{M-1} T_{r}(z) X\left(W_{M}^{r}\right)
$$

where $W_{2 M}=\mathrm{e}^{(-j \pi / M)}, M$ is no. of channels, $T_{0}(z)$ is a distortion transfer function (determines overall amplitude distortion to input signal) and $T_{r}(z)$ is an aliasing distortion transfer function. In perfect reconstruction output signal is same as input except some delay i.e. $y[n]=x\left[n-n_{0}\right]$ for some integer $n_{0}$.

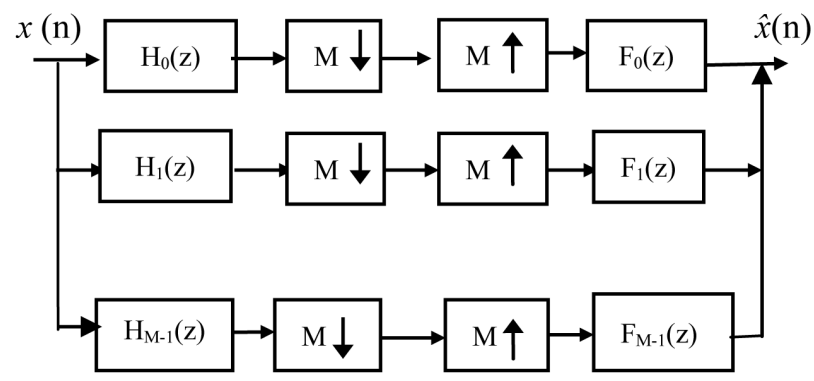

Figure 1. M-channel maximally-decimated Filter bank. 
In cosine modulated filter bank (CMFB) impulse response of all the analysis and synthesis filters generated from the prototype filter $h(n)$ via cosine modulation, can be expressed as follows

$$
\begin{aligned}
& h_{k}(n)=2 h(n) \cos \left((2 k+1) \frac{\pi}{2 M}\left(n-\frac{N-1}{2}\right)+(-1)^{k} \frac{\pi}{4}\right), \\
& f_{k}(n)=2 h(n) \cos \left((2 k+1) \frac{\pi}{2 M}\left(n-\frac{N-1}{2}\right)-(-1)^{k} \frac{\pi}{4}\right) .
\end{aligned}
$$

For $k=0,1, \cdots,(M-1)$ and $n=0,1, \cdots, N-1$. The length $N$ of $H_{k}(z)$ and $F_{k}(z)$ are the same and multiple of $2 M$ to satisfy linear phase property in prototype filters.

Design criteria for effective filter bank are:

\subsubsection{NPR Condition}

In $M$-band cosine modulated filter bank perfect reconstruction conditions [10] for the impulse response of the prototype filter $h[n]$ is

$$
2 \sum_{r=0}^{2 m-2 k-1} h(n+r M) h(n+r M+2 k M)=\delta(k)
$$

where $0 \leq k \leq E\left\lfloor\frac{N}{2 M}\right\rfloor, \quad \delta(k)=\left\{\begin{array}{ll}1, & k=0 ; \\ 0, & k \neq 0 .\end{array}, \quad n=0, \cdots, N\right.$ and $\lfloor E[n]\rfloor$ is the integer part of $n$.

The near perfect reconstruction condition measure is expressed as

$$
\mathrm{NPRV}=\sum_{k=0}^{k=E\left\lfloor\frac{N}{2 M}\right\rfloor}\left(\delta(k)-2 \sum_{r=0}^{N-2 M k} h(r) h(2 M k+r)\right)
$$

\subsubsection{Coding Gain}

A widely accepted measure of coding performance is the Coding Gain (CG) which measures the energy concentration capability of filter banks. By modeling a natural image as a one-dimensional Markovian source with a correlation factor $\rho=0.95$ and by assuming uncorrelated quantization errors, Katto and Yasuda [15] derived a filter dependent coding gain given by:

$$
\mathrm{CG}(\rho)=10 \log _{10}\left(\prod_{k=0}^{M-1}\left(A_{k} B_{k}\right)^{\frac{1}{\alpha_{k}}}\right)
$$

where: $A_{k}=\sum_{i} \sum_{j} h_{k}(i) h_{k}(j) \rho^{|j-i|}, \quad B_{k}=\sum_{i} g_{k}(i)^{2}$ and $h_{k}$ and $f_{k}$ are the $k^{\text {th }}$ analysis and synthesis filter of the $M$-channel uniform filter bank $\alpha_{k}$ is the corresponding subsampling ratio, and $\rho$ is the correlation factor.

\subsubsection{Frequency Selectivity}

As suggested in [7], for high quality cosine modulated filter banks stop band mean energy of the prototype filter should be minimum and the magnitude distortion should be completely cancelled. Aliasing level in Pseudo CMFB can be kept small by keeping the attenuation large for $\omega \geq \frac{\pi}{M}$. The stopband energy can be evaluated by the following expression

$$
E_{s}=\frac{1}{\pi-\omega_{s}} \int_{\omega_{s}}^{\pi}\left|H\left(\mathrm{e}^{\mathrm{j} \omega}\right)\right|^{2} \mathrm{~d} \omega
$$

where $\omega_{s}$ is the stopband edge of the prototype filter.

\subsubsection{Leakage Free}

In image coding, dc leakage free condition does not yield significant improvements in image quality [16]; how- 
ever, to minimize the checkerboard and ringing artifacts dc leakage in subbands other than the lowest one is desired. DC-leakage free property in the cosine modulated filter bank can be expressed as

$$
h^{t} c(\omega)=0, \quad \forall \omega=\frac{(2 k+1) \pi}{2 M}, 1 \leq k \leq M-1
$$

\section{A Multiobjective Genetic Algorithm for the Design of NPR Cosine Modulated Filter Bank}

For optimizing a number of conflicting objective functions simultaneously, multiobjective optimization techniques are used. These techniques provide Pareto-optimal solutions instead of unique optimal solution. These solutions are optimal in the sense that no other solutions in the parameter space are superior to them when all the objective functions are considered [17]-[19].

A multiobjective optimization can be stated mathematically as the minimization problem to minimize

$$
y=f(x)=\left[f_{1}(x), f_{2}(x), \cdots, f_{M}(x)\right]^{\mathrm{T}}
$$

where $x=\left[x_{1}, x_{2}, \cdots, x_{N}\right]^{\mathrm{T}} \in X$ and $y=\left[y_{1}, y_{2}, \cdots, y_{M}\right]^{\mathrm{T}} \in Y$.

Vectors $x$ and $y$ represent the independent variables and the corresponding values of the individual objective functions respectively; on the other hand, $X$ and $Y$ are called the solution space and objective space, respectively.

The notion of Pareto-optimality is an important concept for multiobjective and explained in terms of a "dominance relation" as a solution $x_{i}$ is said to dominate another solution $x_{j}$ if the following conditions hold:

$$
\begin{array}{ll}
f_{k}\left(x_{i}\right) \leq f_{k}\left(x_{j}\right) & \text { For all } k=1,2, \cdots, M ; \\
f_{k}\left(x_{i}\right)<f_{k}\left(x_{j}\right) & \text { For all } k=1,2, \cdots, N .
\end{array}
$$

If a solution is not dominated by any other solutions among the entire objective functions than that solution is said to be a nondominated solution. The solutions that are nondominated regarding the entire parameter space are called Pareto-optimal solutions.

In our design, we search prototype filter $h$ that minimize the three individual objective functions, namely, the energy of the filter stopband, near perfect reconstruction measure and the coding gain. To confine the search process in a feasible solution space, we impose dc leakage free condition as constraint. We have to determine only half of the coefficients since the remaining coefficients are obtained, by applying symmetry.

Our multi-objective optimization problem is formulated as follows:

$$
\min / \max _{h_{0}}\left(\operatorname{objf}_{1}, \operatorname{objf}_{2}, \operatorname{objf}_{3}\right) \text { and }\left\{\begin{array}{l}
\operatorname{objf}_{1}=1 / \mathrm{CG}^{2} ; \\
\operatorname{objf}_{2}=E_{s} ; \\
\operatorname{objf}_{3}=E_{t} .
\end{array}\right.
$$

Subject to the constraint: $h^{t} c(\omega)=0$

In our case, a set of coefficients of the analysis filter bank $(h)$ are treated as chromosomes which are optimized by the constrained multi-objective genetic algorithm (C-NSGA) to obtain a set of filter banks that minimize all the prescribed objective functions with a satisfactory level. This algorithm is based on the NSGA approach [12].

\section{Case Study}

\subsection{Filter Design Examples}

Filter banks designed using non dominated sorting genetic algorithm here use some important parameters i.e. real valued filter coefficients for chromosome construction, crossover operator with distribution index of 20 and probability of 0.9 , a polynomial mutation of distribution index 20 and probability of 0.01 are applied [12] [13]. The population size is set to 100 to find Pareto optimal solutions. Chromosomes of the initial population of filter coefficients are set as $h_{0}=[h(0), \cdots, h(N)]^{t}$ obtained by setting the starting vector as $h(N-1) / 2=(1 / \sqrt{2})$ 
else are equal to zero, satisfying the unit energy constraint between $[-1,1]$. NPR condition is set by keeping the distortion to $10^{-5}$. The maximum generation $\left(G_{\max }\right)$ is set to 2000 generations. The programme was run using MATLAB 7.8 and the obtained coefficients were used for designing NPR cosine modulated filter bank. The amplitude response of the designed filter banks for 8 channel with 16 and 24 taps, with and without dc leakage condition are plotted in Figure 2 and Figure 3. The result of response indicates the superiority of the longer tap filters as the stopband attenuation increase with the increase in no. of taps. Secondly, incorporating dc leakage deteriorate the response of the filter but is good from the image coding point as dc leakage free condition reduce the aliasing errors and all the subband filters except the lowpass one, has at least one zero at $Z=1$ and thus all other bandpass and highpass filters are dc transmission free. Both the filter banks are designed giving highest priority to coding gain amongst the multiple objectives. The $8 \times 16$ CMFB in design Example I, with dc leakage (optimized for pure coding gain); attain $9.3749 \mathrm{~dB}$, which almost equals the coding gain of optimal biorthogonal systems. However, applying dc leakage free constraints on the filter coefficients ensure a high level of perceptual performance in image coding. In design example II, the $8 \times 24$ case, our CMFB achieves an improvement of $0.001 \mathrm{~dB}$ in coding gain. CMFB's with filter length 16 and 24 are shown in Figure 2 and Figure 3. Increasing the length only improves the coding gain marginally, it helps in the case of stopband attenuation (where longer filters are always beneficial), which result in more complexity in design and implementation.

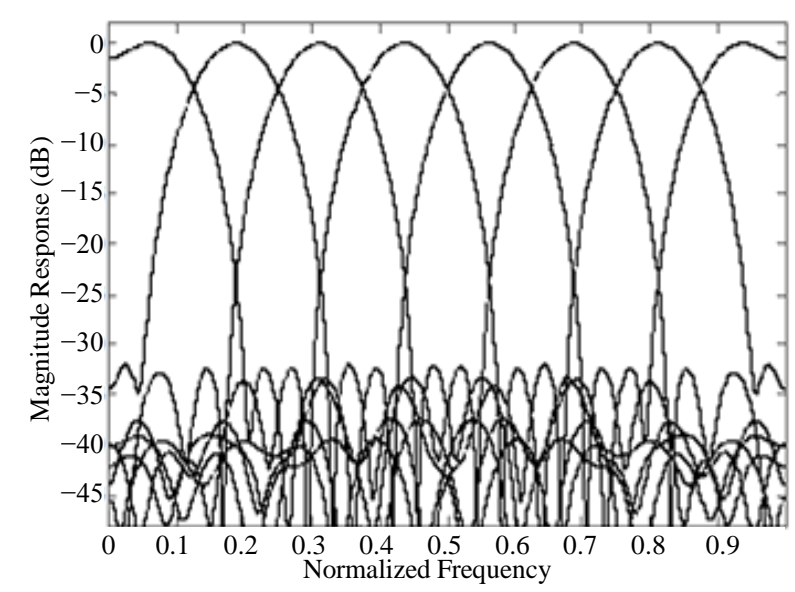

(a)

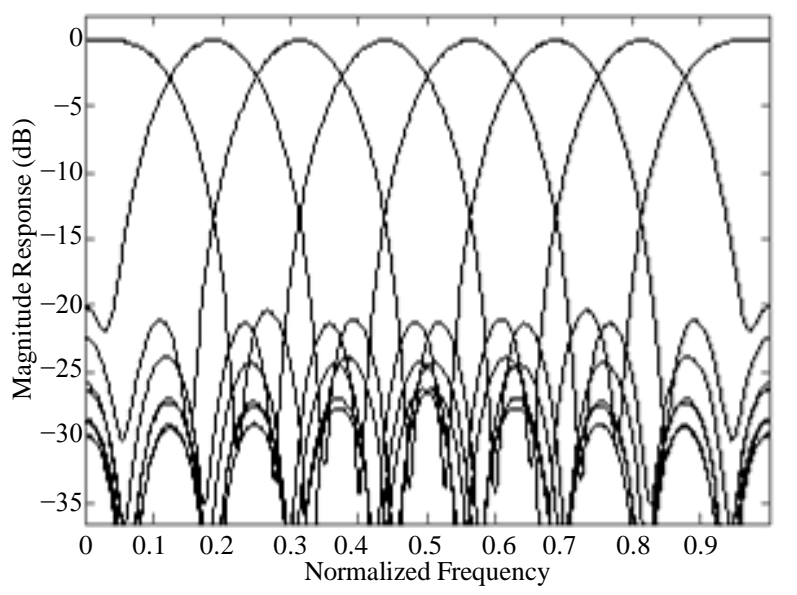

(b)

Figure 2. Magnitude Response of the NPR Cosine modulated filter bank with dc leakage free constraint for (a) $8 \times 16$; (b) 8 $\times 24$.

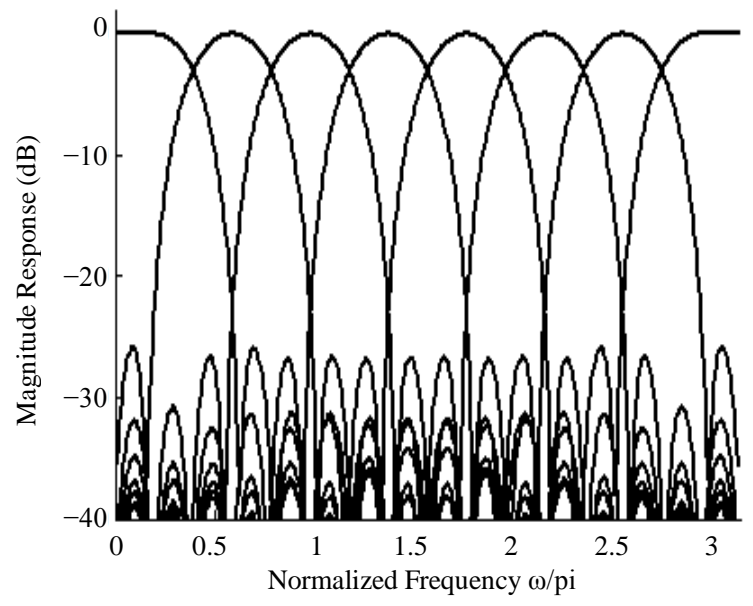

(a)

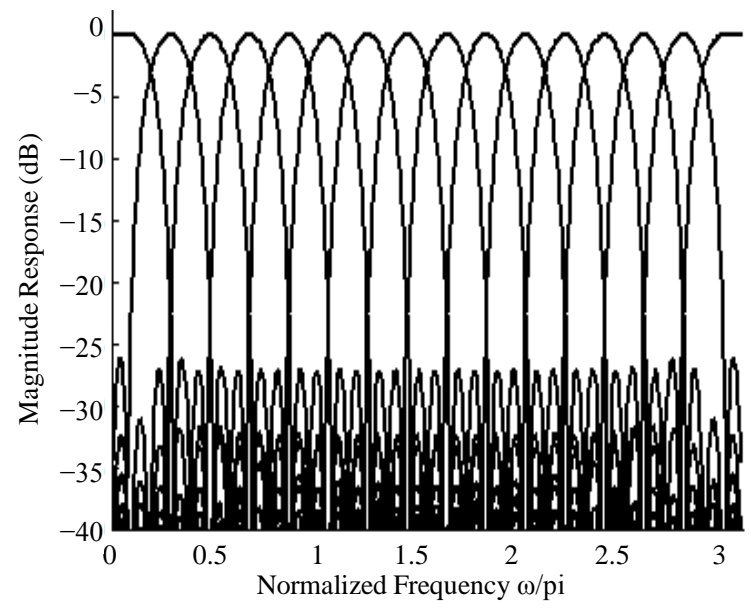

(b)

Figure 3. Magnitude response of the NPR pseudo QMF filter bank with DC leakage constraint designed using multiobjective optimization method for (a) $8 \times 32$; (b) $16 \times 64$. 
Magnitude response of 8 Channel and 16 Channel NPR Pseudo QMF filter banks with 32 and 64 taps respectively, with dc leakage free constraint, designed using multiobjective optimization method, have been shown in Figure 3(a) and Figure 3(b) respectively. Although stopband attenuation is almost same for both the filter banks, clarity of magnitude response shows that the designed filter bank have least dc leakage with coding gain $8.8872 \mathrm{~dB}(8 \times 32)$ and $9.03632 \mathrm{~dB}(16 \times 64)$.

\subsection{Application in Image Coding}

The performance of our designed Cosine Modulated Filter Bank's is evaluated through a progressive block based EZW image coder [20] merely replacing DCT by designed filter bank. In all simulations, we employed a 2 level decomposition of the 8-channel filter bank (1-D transform) separable implementation and symmetric extension at the edges. The coder is chosen to encode the transform coefficients because it provides some of the best performances of any image codec by using the zero tree approach for block based transform and generates an embedded coding which can be helpful in the reconstruction of images at different bitrates.

The images chosen for the coding experiments are Barbara and Boat. Both of them are standard, well known $512 \times 5128$ 8-bit gray-scale test images. The objective distortion measure is the popular peak signal-to-noise ratio (PSNR)

$$
\text { PSNR }=10 \log _{10}\left(\frac{255}{\mathrm{MSE}}\right) \mathrm{dB}
$$

where, MSE denotes the mean squared error. Filter banks $8 \times 16$ and $8 \times 24$ were included in the tests, and both were optimized for maximum coding gain. Reconstructed images "Boat" and "Barbara" using designed filter bank $(8 \times 16)$ and $(8 \times 24)$ are shown in Figure 4(b), Figure 4(c) and Figure 5(b), Figure 5(c) respectively.

It is clear that longer filter banks with more no. of taps have the best characteristics with increase in PSNR. In this context, it is important to note that the filter length cannot be arbitrarily increased in order to increase PSNRs and achieve better frequency selectivity because long filters cause a ringing around edges artefacts when high frequency subband are coarsely quantized. Reconstructed images are better in visual quality as the frequency selectivity improves and overall distortion reduced, however, as the no. of taps increased artifacts become pronounced and PSNR decreases as the bit rates further reduced.

As shown in Figure 4 and Figure 5 for both the images performance of the designed CMFB is good as in low bit-rate coding image is reconstructed using only nonzero coefficients which are few in number and are very concentrated in lowest frequency component (lowpass filter output). Even though coding gain become lower, inclusion of dc leakage free condition leads to smooth reconstructed image as details are preserved.

Objective results for improvement in performance are shown in Table 1 and Table 2. In Table 1 designed filter bank with 24 taps is compared with baseline JPEG using $(8 \times 8$ DCT $)$ as transform and LOT and GENLOT [21]. It is clear that using multiobjective optimization the designed filter bank outperforms as both the coding gain and stopband attenuation are higher as compared other existing filter bank with same no. of parameters.

Results shown in Table 2 indicate the improvement on filter bank performance as the additional criterion of dc leakage free is incorporated in the design. Using multiple objectives our designed filter bank shows a PSNR gain of around $2.5 \mathrm{~dB}$ over a wide range of bit rates at the expense of PR violation.

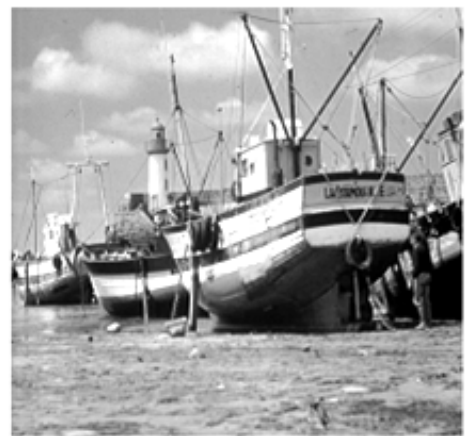

(a)

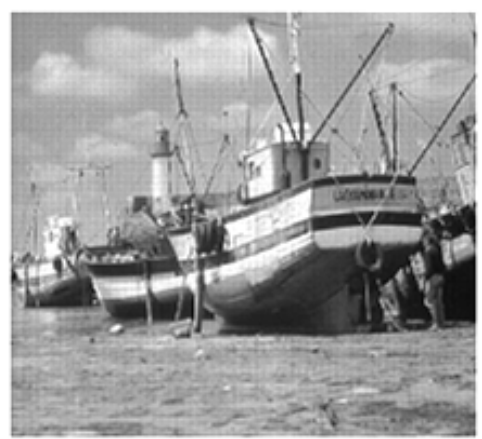

(b)

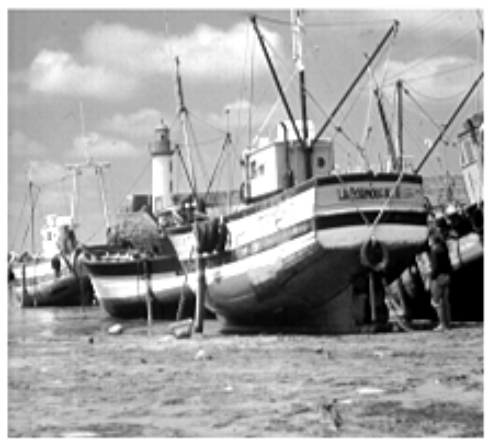

(c)

Figure 4. (a) Original "Boat” Image; (b) Reconstructed image with $(8 \times 16)$ NPR CMFB filter bank; (c) Reconstructed image with $(8 \times 24)$ NPR CMFB filter bank. 


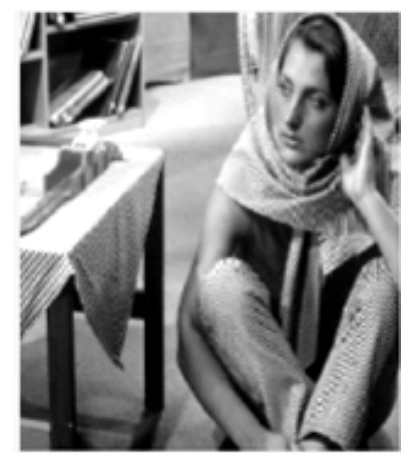

(a)

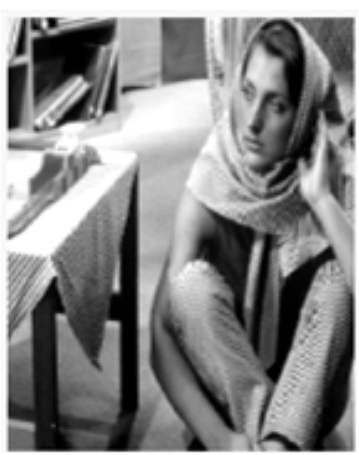

(b)

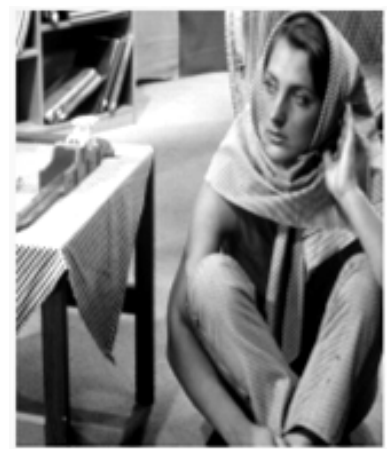

(c)

Figure 5. (a) Original "Barbara" image; (b) Reconstructed image with $(8 \times 16)$ NPR CMFB filter bank; (c) Reconstructed image with $(8 \times 24)$ NPR CMFB filter bank.

Table 1. Comparison of different transform's performance.

\begin{tabular}{ccc}
\hline Transform & Coding Gain & Stopband Attenuation $(\mathbf{d B})$ \\
\hline $8 \times 8$ DCT & 8.83 & 9.96 \\
$8 \times 16$ LOT & 9.22 & 19.38 \\
$8 \times 24$ GenLOT & 9.35 & 23.20 \\
Proposed $(8 \times 24)$ & 9.3749 & 26.026 \\
\hline
\end{tabular}

Table 2. Image coding results for "Barbara” image at 0.25 bpp.

\begin{tabular}{cccccc}
\hline \multirow{2}{*}{$\begin{array}{c}\text { M } \\
\text { No. of channels }\end{array}$} & \multirow{2}{*}{$\begin{array}{c}\text { Length of } \\
\text { prototype filter }\end{array}$} & \multicolumn{2}{c}{ With dc leakage } & \multicolumn{2}{c}{ Without dc leakage } \\
\cline { 3 - 6 } & PSNR & CG & PSNR & CG \\
\hline 8 & 16 & 28.02 & 8.89 & 29.99 & 8.85 \\
8 & 24 & 30.11 & 9.37 & 31.18 & 9.21 \\
8 & 32 & 32.02 & 9.374 & 33.63 & 9.257 \\
\hline
\end{tabular}

\section{Conclusion}

The design of $M$-channel uniform cosine modulated filter banks using genetic algorithm is discussed in this paper. The design problem having multiple objectives is solved using non-dominated sorting algorithm with regularity constraint. Although dc leakage free condition is not an essential requirement for image processing in $M$ channel filter bank as PSNRs don't improve considerably, its imposition identifies infeasible solutions thereby confines the search for the NPR filter banks simultaneously, and improve visual quality of images at low bit rates. From simulation result, it is shown that the algorithm results in lower tap filters with high stopband attenuation, the least overall distortion and higher coding gain for image compression. Use of progressive image coder based on block-based EZW improves PSNR via reducing blocking and ringing artefacts. The work can be further extended to design of biorthogonal cosine modulated filter banks for image coding application.

\section{References}

[1] Esteban, D. and Galand, C. (1977) Application of Quadrature Mirror Filters to Split Band Voice Coding Schemes. Proceedings of IEEE International Conference on Acoustics, Speech, and Signal Processing, ASSP, May 1977, 191195.

[2] Smith, M.J.T. and Eddins, S.L. (1990) Analysis/Synthesis Techniques for Sub-Band Image Coding. IEEE Transactions on Acoustics, Speech and Signal Processing, ASSP-38, August 1990, 1446-1456. http://dx.doi.org/10.1109/29.57579

[3] Woods, J.W. and O’Neil, S.D. (1995) Sub-Band Coding of Images. IEEE Trans. Acoustics Speech, PTR, Englewood Cliffs.

[4] Vatterli, M. and Kovacevic, J. (1995) Wavelet and Subband Coding. Prentice Hall PTR, Englewood Cliffs. 
[5] Steffen, P., Heller, P.N., Gopinath, R.A. and Burrus, C.S. (1993) Theory of Regular M-Band Wavelet Bases. IEEE Transactions on Signal Processing, 41, 3497-3510. http://dx.doi.org/10.1109/78.258088

[6] Gopinath, R.A. and Burrus, C.S. (1994) On Cosine-Modulated Wavelet Orthonormal Bases. IEEE Transactions on Image Processing, 4, 162-175. http://dx.doi.org/10.1109/83.342190

[7] Nguyen, T.Q. (1994) Near Perfect Reconstruction Pseudo-QMF Banks. IEEE Transactions on Image Processing, 42, 65-75. http://dx.doi.org/10.1109/78.258122

[8] Lin, Y.P. and Vaidyanathan, P.P. (1995) Linear Phase Cosine Modulated Maximally Decimated Filter Banks with Perfect Reconstruction. IEEE Transactions on Image Processing, 43, 2525-2539. http://dx.doi.org/10.1109/78.482104

[9] Vaidyanathan, P.P. (1993) Multirate Systems and Filter Banks. Prentice Hall, Englewood Cliffs.

[10] Rossi M., Zhan J.Y. and Steenaart, W. (1996) Iterative Constrained Least Square Design of Near Perfect Reconstruction Pseudo qmf Banks. IEEE CCECE, 1996, 766-769.

[11] Jain, A. and Goel, A. (2012) Unconstrained Optimization Method for Design of M Channel Perfect Reconstruction CMFB Filter Bank. Proceedings of the International Conference on Signal, Image and Video Processing, Patna, 13-15 January 2012, 302-308.

[12] Boukhobza, A., Ahmed, T., Bounoua, A. and Taleb, N. (2009) A Filter Banks Design Using a Multi-Objective Genetic Algorithm for Embedded Image Coding Scheme. Proceedings of the 6th International Symposium on Image and Signal Processing and Analysis, Salzburg, 16-18 September 2009, 776-781.

[13] Boukhobza, A., Ahmed, T., Bounoua, A. and Taleb, N. (2009) A Filter Banks Design Using a Multi-Objective Genetic Algorithm for an Image Coding Scheme. Proceedings of the 16th IEEE International Conference on Image Processing, Cairo, 7-10 November 2009, 1933-1936.

[14] Heller, P.N., Karp, T. and Nguyen, T.Q. (1999) A General Formulation for Modulated Filter Banks. IEEE Transactions on Signal Processing, 47, 986-1002. http://dx.doi.org/10.1109/78.752597

[15] Katto, J. and Yasuda, Y. (1991) Performance Evaluation of Subband Coding and Optimization of Its Filter Coefficients. Proceedings of the SPIE Symposium on Visual Communications and Image Processing, 1605, 95-106.

[16] Karp, T. and Mertins, A. (1998) Biorthogonal Cosine-Modulated Filter Banks without DC Leakage. Proceedings of the 1998 IEEE International Conference on Acoustics, Speech and Signal Processing, Seattle, 12-15 May 1998, 14571460.

[17] Deb, K. (1999) Evolutionary Algorithms for Multi-Criterion Optimization in Engineering Design. Proceedings of Evolutionary Algorithms in Engineering and Computer Science (EUROGEN-99), Jyväskylä, 29 May-3 June 1999, 135161.

[18] Deb, K., Pratab, A., Agarwal, S. and Meyarivan, T. (2002) A Fast and Elitist Multi-Objective Genetic Algorithm: NSGA-II. IEEE Transactions on Evolutionary Computation, 6, 182-197. http://dx.doi.org/10.1109/4235.996017

[19] Goldberg, D.E. (1989) Genetic Algorithms in Search Optimization and Machine Learning. Addison-Wesley, Boston.

[20] Xiong, Z., Galerius, O.G. and Orchard, M.T. (1996) A DCT Based Embedded Image Coder. IEEE Signal Processing Letters, 3, 289-290. http://dx.doi.org/10.1109/97.542157

[21] de Queiroz, R.L., Nguyen, T.Q. and Rao, K.R. (1996) The GenLOT: Generalized Linear-Phase Lapped Orthogonal Transform. IEEE Transactions on Signal Processing, 44, 497-507. http://dx.doi.org/10.1109/78.489023 
Scientific Research Publishing (SCIRP) is one of the largest Open Access journal publishers. It is currently publishing more than 200 open access, online, peer-reviewed journals covering a wide range of academic disciplines. SCIRP serves the worldwide academic communities and contributes to the progress and application of science with its publication.

Other selected journals from SCIRP are listed as below. Submit your manuscript to us via either submit@scirp.org or Online Submission Portal.
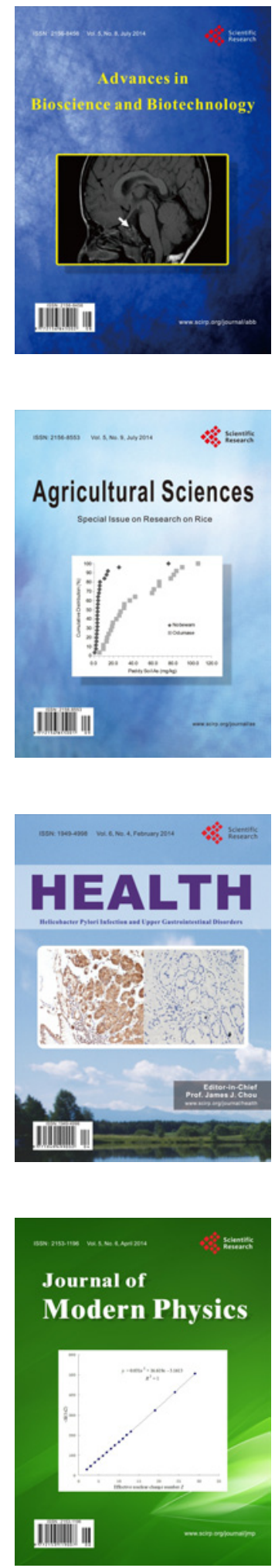
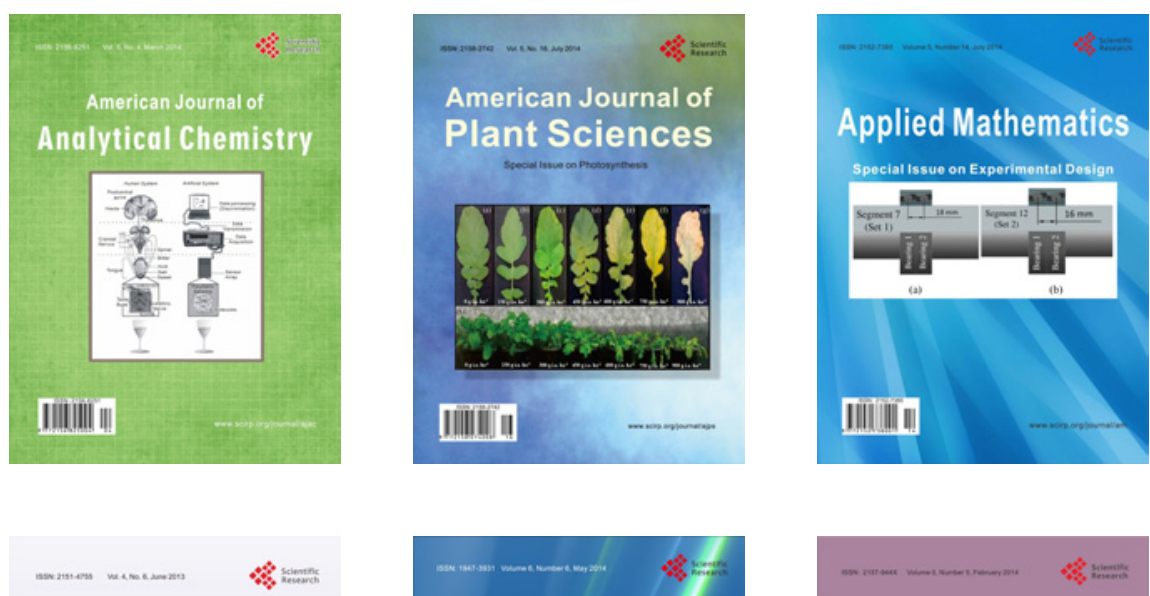

Creative Education
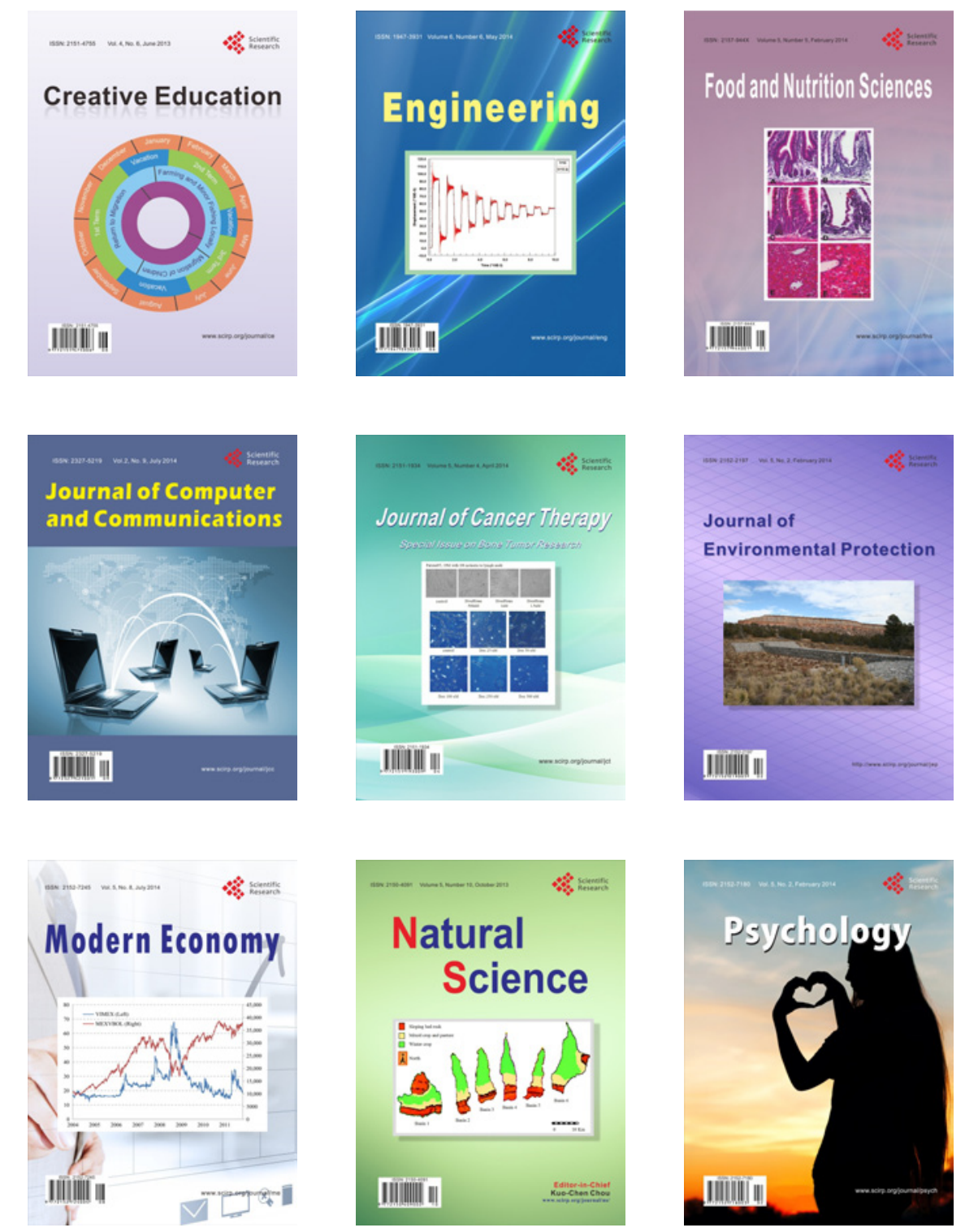